\title{
Brain iron redistribution in mesial temporal lobe epilepsy: a susceptibility-weighted magnetic resonance imaging study
}

\author{
Zhiqiang Zhang ${ }^{1 *}$, Wei Liao ${ }^{1,2}$, Boris Bernhardt ${ }^{3}$, Zhengge Wang ${ }^{1}$, Kangjian Sun ${ }^{4}$, Fang Yang ${ }^{5}$, Yijun Liu ${ }^{6}$ \\ and Guangming Lu'
}

\begin{abstract}
Background: The roles of iron in epilepsy and its pathophysiological significance are poorly understood, especially whether iron levels are abnormal in subcortcal structures. This study aims to demonstrate whole-brain iron alterations and its clinical relevancies in mesial temporal lobe epilepsy (mTLE) in vivo, using susceptibility-weighted magnetic resonance imaging (SWI).

Methods: We studied 62 patients with mTLE and 62 healthy controls. Brain iron concentration was quantified using SWI phase values. Voxel-wise analysis was carried out to compare iron levels between mTLE and controls, and to assess the relationship between altered iron concentration and clinical parameters in MTLE.

Results: Patients with mTLE showed decreases of iron levels in the subcortical structures such as substantia nigra, red nucleus, and basal ganglia. Conversely, iron levels were decreased in the cortex. Subcortical iron levels were negatively correlated to those in the cortex. Moreover, cortical and basal ganglia iron levels were related to clinical variables including epilepsy duration, age at seizures onset, and histories of precipitating factors.

Conclusions: Our SWI findings suggest a redistribution of iron between subcortical and cortical structures in mTLE. The degree of redistribution is affected by both progression of epilepsy and precipitating factors. Investigation on brain iron redistribution offers new insights into the pathogenesis of $\mathrm{MTLE}$, and may be a potential biomarker for monitoring the clinical progression of epilepsy.
\end{abstract}

Keywords: Brain iron, Mesial temporal lobe epilepsy, Susceptibility-weighted magnetic resonance imaging

\section{Background}

Iron is essential for many brain physiological processes ranging from gene expression, neuronal development, enzymatic reactions, dopamine synthesis and electron transport [1-3]. Moreover, abnormal iron levels have been found in many neurological disorders, such as Parkinson's disease [4], Alzheimer's disease [5] and Restless Legs Syndrome [6], suggesting that the measurement of cerebral iron concentration may be a potential biomarker to advance the understanding, diagnosing, monitoring, and treatment of diseases $[2,7]$.

\footnotetext{
*Correspondence: zhangzq2001@126.com; cjr.luguangming@vip.163.com ${ }^{1}$ Department of Medical Imaging, Jinling Hospital, Nanjing University School of Medicine, 305\# Eastern Zhongshan Rd, Nanjing 210002, China Full list of author information is available at the end of the article
}

There is initial evidence for a close link between alterations in brain iron and epilepsy [8-10]. In animal models, injection of ferric or ferrous chloride into neocortical regions or hippocampus has resulted in electrographic and behavioral seizures [9]. The free radicals generated by iron have been shown to attack cell membranes by lipid peroxidation, leading to neuronal damage and ultimately epileptic discharges [11]. In rat model of epilepsy, up-regulated expression of ferritin, an iron storage protein, has been observed in regions particularly vulnerable to cell death [10]. In human patients, observational data on a direct link between iron and epileptogenis is scarce. Increased iron burden, often subsequent to intraparenchymal hemorrhages and trauma, may be associated with an elevated incidence of epilepsy [12,13]. Other indirect evidence for a link between epilepsy and iron comes 
from work on resected tissue, where blood-brain barrier leakage, which may cause local extravasation of blood and release of iron from haemoglobin-containing blood cells, may take place secondary to seizures [14].

The invasive nature of histological approaches to measure iron levels [15] precludes their use in epileptic patients $[16,17]$. The recent development of magnetic resonance imaging (MRI) techniques based on susceptibility-weighted imaging (SWI), offers an accurate in vivo measurement of brain iron deposition [7]. Indeed, phase images of SWI, a high-resolution, 3D, and fully flow-compensated gradient echo sequence, are sensitive to subvoxel magnetic inhomogeneities affected by iron in the forms of haemosiderin, ferritin and deoxyhaemoglobin. It has been shown that SWI and can measure iron levels on the order of just $1 \mathrm{mg} / \mathrm{g}$ tissue in vivo $[7,18]$. While previous studies have used SWI to assess the iron alterations in non-blood brain tissue (non-heme iron) and their clinical relevance in Parkinson disease, Alzheimer Disease and multiple sclerosis $[4,5,19]$. There are to date no data on iron-related SWI changes in epilepsy.

The purpose of the current study was to employ voxelbased SWI analysis to investigate the topography of brain iron alterations in mTLE. (1) Given that the previous studies mostly focus alterations of iron concentrations in epilepsy in the cortical regions [8], the iron alterations in subcortical structures in epilepsy thus still remain poorly understood. In human brain, non-hemo iron is highly concentrated in the globus pallidus (GP), substantia nigra $(\mathrm{SN})$ and red nucleus (RN), and is an essential cofactor in the synthesis of dopamine [20]. Considering the important roles of dopaminergic neurons and the subcortical structures played in mTLE $[21,22]$, it is conceivable that the subcortical iron may be associated with the process of epilepsy. Hence we first assessed phase alterations in the subcortical structures in mTLE, and particularly interested in the relationship between subcortical and cortical phase alterations. (2) Considering that there is currently lack of clinical significances of iron alterations in epilepsy, we correlated brain phase values with clinical variables of epileptic patients, in order to explore the possible roles of brain iron played in the pathogenesis and progression in mTLE.

\section{Methods \\ Participants}

We recruited 62 consecutive adult patients with mTLE who had received clinical treatments in Jinling Hospital. Demographic and clinical data are detailed in the Table 1. MTLE diagnosis and lateralization of the seizure focus were determined by a comprehensive evaluation, including seizure history and semiology, neurological examination, diagnostic MRI, and EEG records in all patients. Patients were retrospectively selected if they satisfied the following criteria: (i) All patients were young and middleaged adults. Patients younger than 18 yrs, or older than 50 yrs were excluded. (ii) Patients with other identifiable structural MRI abnormalities than hippocampal sclerosis, such as cortical dysplasia, vascular malformation or tumor were excluded. (iii) Pathogenesis of mTLE. Twenty-three patients with prior history of febrile convulsion, 14 patients with intracranial infections and 25 patients without pathogenic history were involved; patients with the other pathogenesis, such as head trauma and poisoning were excluded. Patients were compared to 62 age- and gendermatched controls, recruited from the staff of Jinling Hospital. Controls did not suffer from neurological or psychiatric disorders at the time of the study.

This study was approved by the Medical Ethics Committee in Jinling Hospital, Nanjing University School of Medicine (Reference number: 2012GJJ-055). All examinations were carried out under the guidance of the Declaration of Helsinki 1975. Written informed consent was obtained from all the participants.

\section{MRI data acquisition}

MRI data were collected on a 3 T scanner (MAGNETOM Trio, Siemens Healthcare, Erlangen, Germany) equipped

Table 1 Demographic and clinical information of patients with mTLE and healthy controls

\begin{tabular}{|c|c|c|c|c|c|c|c|}
\hline Groups & Men & Age & Duration & Onset age & Seizure frequency & History & Hippocampal sclerosis \\
\hline Controls $(n=62)$ & 34 & $27.5 \pm 8.1(18-48)$ & None & None & None & None & None \\
\hline \multirow[t]{3}{*}{$\operatorname{LTLE}(n=31)$} & 18 & $26.1 \pm 7.6(18-48)$ & $11.8 \pm 8.8(1-29)$ & $14.4 \pm 8.9(1-46)$ & $3(2-150)$ & FC: 13 & LHS: 27 \\
\hline & & & & & & I: 8 & \\
\hline & & & & & & $\mathrm{NH}: 10$ & BHS: 4 \\
\hline \multirow[t]{3}{*}{$\operatorname{RTLE}(n=31)$} & 16 & $28.8 \pm 8.4(18-48)$ & $10.5 \pm 8.3(1-38)$ & $18.4 \pm 9.8(1-37)$ & $5(0.5-90)$ & FC: 10 & RHS: 30 \\
\hline & & & & & & $\|: 6$ & \\
\hline & & & & & & $\mathrm{NH}: 15$ & BHS: 1 \\
\hline
\end{tabular}

LTLE: left temporal lobe epilepsy; RTLE: right temporal lobe epilepsy.

Age, duration of epilepsy and age at onset are presented in mean \pm SD (range) years. Seizure frequency is given as median (range) of seizures/mo. II: intracranial infection; FC: febrile convulsion; NH: no prior history.

Definitions: epilepsy duration: period from the time of the first independent seizures to the time of MRI scan; ages of seizures onset, ages at the first independent seizures onset; and seizure frequency, seizures frequency over the recent one-year. 
with an eight-channel, phase-array head coil. Whole-brain high-resolution SWI data including phase images were obtained parallel to the anterior-posterior commissural line using a 3-Dimensional gradient-echo sequence (TR/ TE: $28 / 20 \mathrm{~ms}$; flip angle: $15^{\circ}$; slices: 48 ; field of view: $240 /$ $240 \mathrm{~mm}$; matrix: $448 \times 358$ and oversampling: $16.7 \%$ ). SWI acquisition generated phase images, magnitude images, SWI with overlapping phase images on magnitude images and SWI with minimal intensity project (mIP) reconstruction [18]. These SWI images were all online processed automatically on a workstation using the Syngo VB17 software (Siemens Medical Solution), and phase images were high-pass filtered $(64 \times 64$ low spatial frequency kernel). Moreover, we obtained high-resolution T1-weighted anatomical images using a 3D Magnetization Prepared Rapid Acquisition Gradient-echo (MPRAGE) sequence $\left(\mathrm{TR} / \mathrm{TE}=2300 \mathrm{~ms} / 2.98 \mathrm{~ms}\right.$, FA $=9^{\circ}$, matrix $=$ $256 \times 256, \mathrm{FOV}=256 \times 256 \mathrm{~mm}^{2}$, slice thickness $=1 \mathrm{~mm}$ ). Moreover, coronal T1 $(\mathrm{TR} / \mathrm{TE}=280 / 2.5 \mathrm{~ms}$; FOV $=230 \times$ $230 \mathrm{~mm}^{2}$, slice thickness $=4 \mathrm{~mm}$, no gap, 18 slices) and T2 FLAIR $\left(\right.$ TR/TE $=8000 / 93 \mathrm{~ms}$; FOV $=230 \times 230 \mathrm{~mm}^{2}$, slice thickness $=4 \mathrm{~mm}$, no gap, 18 slices) images were collected to measure hippocampal volume and detect hippocampal signal abnormalities.

\section{Data preprocessing}

\section{Intensity preprocessing of the phase images}

Firstly, the unwarpped and high-pass filtered $[7,18]$ phase images were linearly scaled to a phase value range of $\pi$ to $-\pi$. Subsequently, voxels with positive phase value (i,e, those ranging between 0 and $\pi$ ) were removed. This step was performed to exclusively measure iron content in the brain, because it is well-known that iron in tissue has a negative-phase effect [18]. Secondly, we automatically identified brain veins showing dark signal intensity in the MIP images individually, and replaced the corresponding voxels in the phase images by mean global signal intensity of the phase image. Following these preprocessing steps, the phase values are believed to represent the content of the non-hemo iron deposition [18].

\section{Spatial preprocessing of the phase images}

Spatial preprocessing of the phase images was performed using SPM8 for Matlab. Data of patients with left-sided mTLE and controls were left-right flipped. Phase images of all subjects were linearly co-registered to their highresolution T1-weighted images, then the affine matrix by normalizing the 3D anatomic images to a T1 template with Montreal Neurological Institute (MNI) 305 coordination was written to the phase images after intensity processing. Normalized phase images were further re-sampled to a resolution of $1.5 \times 1.5 \times 1.5 \mathrm{~mm}$, and smoothed with a $4 \mathrm{~mm}$ Full-width-at-half-maximum Gaussian kernel to increase the signal-noise ratio (Figure 1). Prior to group analyses, subject-level voxel-wise phase value maps were standardized into subject-level $Z$-score maps (i.e., by subtracting the mean voxel-wise phase obtained for the entire brain, and then dividing by the standard deviation). The standardized phase can improve the subsequent statistical analyses on group-level phase measures [23].

\section{Voxel-based analyses of SWI phase values in MTLE}

Phase images of all subjects were preprocessed for the removal of heme-iron confounds and spatially normalized to a standard stereotaxic space. Voxel-based analyses were carried out using SPM8 (http://www.fil.ion.ucl.ac.uk/spm) for Matlab (The Mathworks, Natick, MA).

\section{Group comparison}

We first observe the topological pattern of iron distribution in human brain by performing one-sample t-test on SWI phase values of all subject groups. We subsequently applied two-sample $t$-test on SWI phase values between patients and controls to map the topological pattern of altered brain iron in mTLE. The gray matter (GM) volume of each subject extracted from voxel-based morphometric analysis, was regressed voxel-wisely. Moreover, for validating the result of voxel-based analysis, we additionally performed ROI-based comparison analyses (See Additional file 1: Figure S1).

\section{Correlation analysis between regions of iron changes}

We performed a post-hoc correlation analysis to study the relationship between the subcortical showing altered phase values and rest of the brain. The bilateral RN and $\mathrm{SN}$, which showed most intensively decreased phase values in patients with mTLE (see the Results for details), were combined and selected as seed region. A voxel-based correlation analysis measuring the covariance of phase values across subjects was conducted, which can detect the regions whose phase values were correlated with those in the seeds. The correlation patterns of the patients and controls were compared using a classic interaction linear model [24]. For each subject group, the phase values in the regions of $\mathrm{RN}+\mathrm{SN}$ (seed region for correlation maps), cortex and basal ganglia were extracted out. The region of cortex and basal ganglia were defined according to the correlation map of the patients.

\section{Subgroup assessment of effect of precipitating factors}

Within the patient group, we applied a one-way analysis of variance (ANOVA) to compare patients with a prior history of febrile seizures $(n=23)$, patients with intracranial infections $(\mathrm{n}=14)$, and patients with no overt history $(\mathrm{n}=15)$. The ANOVA analysis was assumed to find the specific iron distribution patterns in MTLE with different precipitating factors. 


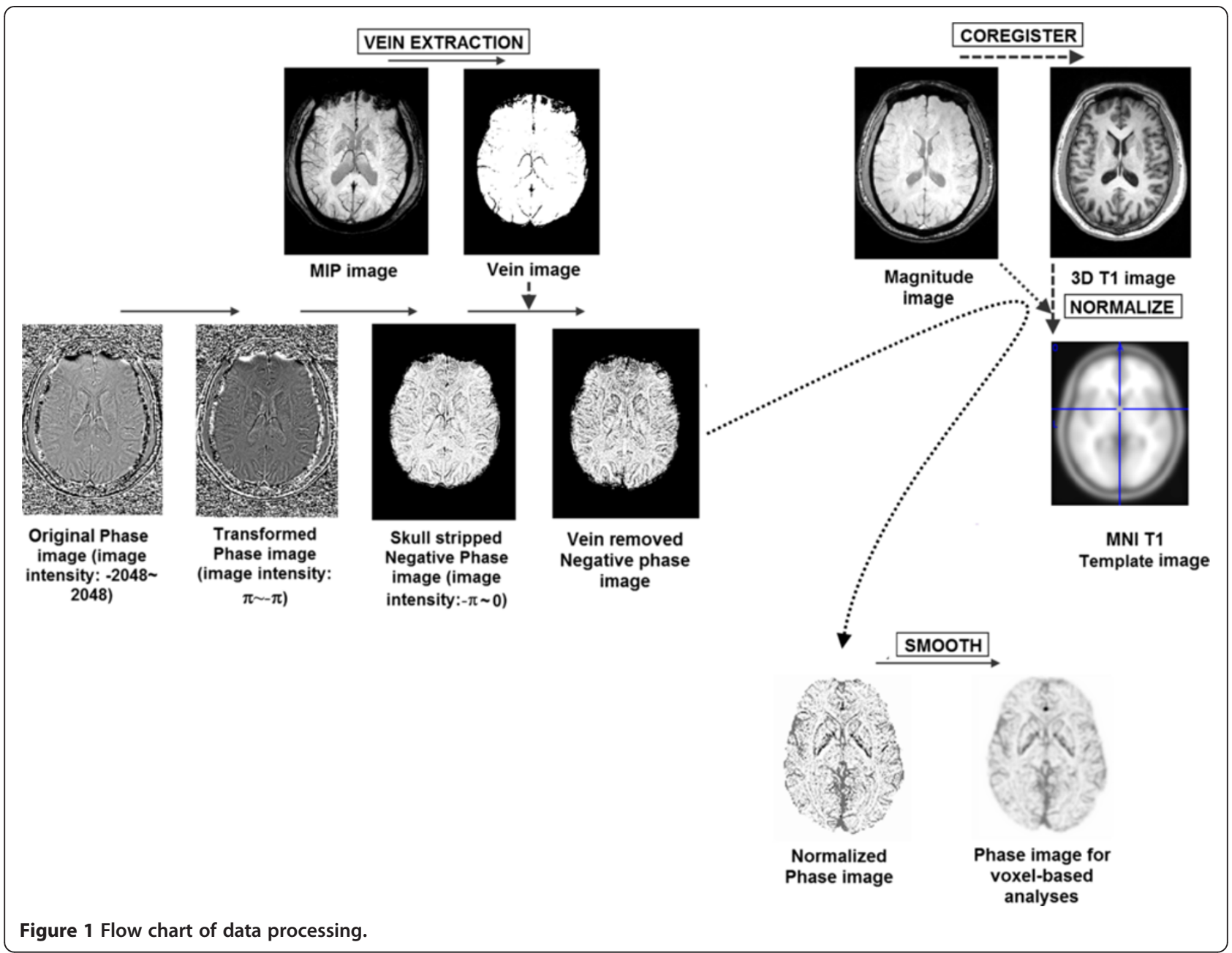

Correlation analyses between the phase values and clinical variables in $m T L E$

Voxel-based correlation analyses were performed to highlight regions of correlation between SWI phase values and the clinical variables epilepsy duration, age at seizures onset, and seizure frequency, (as defined in Table 1). Because epilepsy duration was negatively correlated with age at seizure onset $(r=-0.607, p<0.001)$ in our patients, these two clinical variables were regarded as covariates each other in the voxel-based correlation analyses. During the above voxel-based analyses, subject age and gender were included in the model as nuisance covariates [25].

\section{Correction for multiple comparisons}

We used the false discovery rate $($ FDR, $P<0.05)$ procedure to correct for multiple comparisons during all voxel-based analyses [26].

\section{Results}

Patterns of abnormal SWI-phase in MTLE relative to controls

One-sample t-tests revealed topological patterns of phase values. Apparently, the subcortical structures including the globus pallidus (GP), substantia nigra (SN) and red nucleus (RN) showed lower phase values in contrast to the white matter and cortex. Analyzing differences in global mean SWI phase, there was no statistical difference $(t=1.036, p=0.328)$ between patients (mean \pm std: $-0.047 \pm 0.009)$ and controls $(-0.048 \pm$ $0.009)$. On the other hand, voxel-wise group comparisons decreases in SWI phase (i.e., increased iron concentration) in mTLE in widespead cortical networks including frontal, temporal, and occipital cortical areas $(P<0.05$, FDR correction), as well as increased phase (i.e., decreased iron concentration) in subcortical structures such as the bilateral internal globus pallidus (GPi), putamen (PUT), RN and SN (Figure 2). ROI-based 

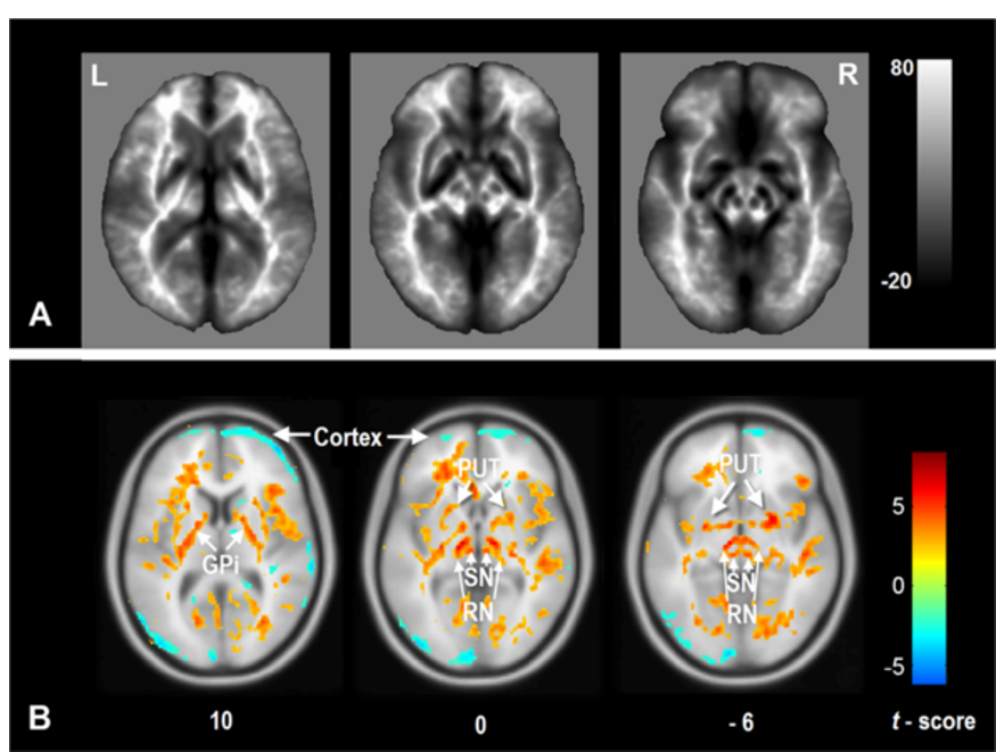

Figure 2 Comparison of the whole brain phase values between the patients with mTLE and healthy controls. A: One-sample t-test for processed phase images in all subjects. It is apparent that the sub-cortical nuclei, including the globus pallidus, substantia nigra and red nucleus showed lower phase values (or higher iron concentration) than the other brain structures. B: Two sample t-tests analyses revealed increased iron concentration in mTLE in the neocortex, and decreased iron concentration in the subcortical structures. SWI phase value were decreased in multiple cortical regions in mainly frontal, temporal and occipital lobes; increased SWI phase was found in sub-cortical structures including the bilateral GPi, RN and SN. Finding have been thresholded at $P<0.05$, FDR correction. The results are shown by overlying on MNI 305 template image. Abbreviations: L: left; R: right; PUT: putamen; GPi: internal globus pallidus; SN: substantia nigra; RN: red nucleus.

comparison analyses repeated the VBA results (See Additional file 1: Table S1).

\section{Cortico-subcrotical iron level correlations}

In mTLE, voxel-wise correlation analysis seeding from the $\mathrm{RN}$ and SN confirmed and extended the patterns seen in the group analysis. Indeed, we observed widespread positive correlations of RN/SN with the other basal ganglia such as PUT and GPi, and negative correlations with neocortical regions. This result indicates that patients with high iron levels in SN and RN also tend to have high iron levels in PUT and GP, but low iron in cortical areas. This negative correlation pattern was not found in controls (Figure 3 and Additional file 1: Table S2).

\section{Effect of precipitating factors}

We observed different patterns of iron deposition alterations in patients with different precipitating factors (Figure 4 and Table 2). Compared to the subgroup of intracranial infection, the subgroups of febrile seizures and no overt history both showed higher phase values (i.e., lower iron level) in the bilateral PUT, RN and SN. However, there were no significant alteration of phase values when comparing subgroup of febrile seizures and that of no overt history.

\section{Effects of clinical variables}

We observed a negative correlation between SWI phase in cortical regions and a majority of subcortical structures (including bilateral PUT, external globus pallidus [GPe], $\mathrm{SN}$ and $\mathrm{RN}$ ) to duration of epilepsy. These findings indicate lower iron levels in patients with long-withstanding seizures in these regions. In the GPi, on the other hand, we observed a positive correlation, indicating progressive iron increase.

Analyzing effects of seizure onset, subcortical finding resembled the pattern seen in the previous analysis of duration of epilepsy. Whereas, we did not observe any significant correlation in the cortical regions. We did not observe any correlation to seizure frequency in the brain regions as described above (Figure 5 and Table 3 ).

\section{Discussion}

Measuring the phase values of MRI-SWI, this study provided novel insights into the whole brain changes of iron state in patients with mTLE in vivo. We observed increased cortical brain iron deposition in the patients, together with decreases in the subcortical structures such as the PUT, GP, SN and RN. Strikingly, the negative correlation of phase values between the cortical and subcortical structures suggests that these iron alterations in the brain structures may result from redistribution of 

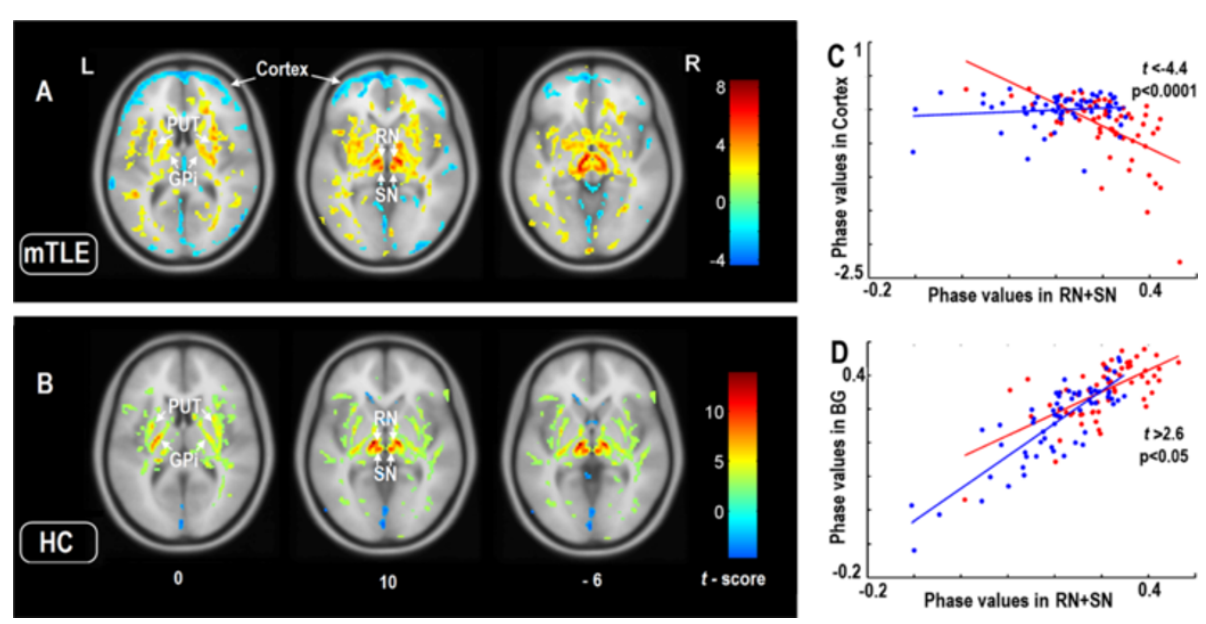

Figure 3 Correlation analysis of cortical and subcortical iron levels. Covariance analysis between cortical and subcortcial SWI phase values in patients with mTLE (A) and healthy controls (B). Seeds were based on the peak coordinates of group difference in the SN and RN (see Figure 1). In mTLE, seed SWI phase was positively correlated to multiple subcortical regions, and negatively correlated with neocortical regions. In healthy controls, subcortical positive correlations were more restricted and there was no negative correlation between the seed regions and cortical areas. Findings have been thresholded at $P<0.05, F D R$ correction. The results are shown by overlying on MNI 305 template image.The right panels illustrate the differences of interregional correlation of phase values in patients and controls. (C): Interregional correlations between the seed region $(S N+R N)$ and the cortex. The phase values in the $S N+R N$ was negatively correlated those in the cortex $\left(r=-0.59, p=4.92 \times 10^{-9}\right)$ in the patient group; but no significantly correlation was found in the control group ( $r=0.12, p=0.34)$. The correlations were different between the patients and controls $(t=-4.44, p<0.0001)$. (D): Interregional correlations between the seed region $(S N+R N)$ and the basal ganglia. The phase values in the $S N+R N$ was positively correlated those in the basal gangila in both patient $\left(r=0.66, p=4.92 \times 10^{-9}\right)$ and control groups $(r=0.85$, $p=2.47 \times 10^{-18}$ ). In the plots, phase values and regression lines are shown in red for the patients and in blue for controls. The correlations were different between the patients and controls $(t=2.61, p<0.05)$. The comparisons of correlations were performed using a classic interaction linear model. Abbreviations: L: left; R: right; mTLE: mesial temporal lobe epilepsy; HC: healthy controls; PUT: putamen; GPi: internal globus pallidus; SN: substantia nigra; RN: red nucleus.

brain iron stores in epilepsy. Clinical analyses further implicate that the changes of cerebral iron deposition are relevant with, and may play important roles in the progression and pathogensis of mTLE.

\section{Pattern of altered brain iron distribution in mTLE}

In cortical regions, we observed an increased cortical iron deposition in mTLE, which is consistent with the findings in previous animal and clinical research [10,12,14]. Moreover, our result for the first time provided the panorama of increased iron in the diffuse cortical structures. Increased levels of brain iron have previously been interpreted to be a causative factor of epilepsy due to the damaging effect of iron [8]. Increased iron can lead to lipid peroxidation and then affects protein function, which is associated with increased excitation (increased extracellular glutamate) and decreased inhibition (decreased function of $\mathrm{GABA}_{\mathrm{A}}$ receptor) of neurons in epilepsy $[1,3,6,8,27]$.

Conversely to the findings in the cortical regions, we observed decreased iron levels in the basal ganglia subcortical nuclei, including the bilateral PUT, GP, SN and RN in the patients. This novel finding revealed that these subcortical nuclei, which containing the highest levels of nonhemo iron in the brain, are sensitive to the disturbance of iron metabolism in epilepsy. Basal ganglia subcortical nuclei may contribute to the patho-physiological process of epilepsy through their role in unilateral dystonic posturing seizure and inhibition of seizure activity [21,28,29]. $\mathrm{SN}$ and RN play important roles in propagation of seizure activity [22]. Given that the subcortical structures are densely innervated by dopaminergic pathways, and iron is an essential cofactor in the synthesis of dopamine [20], our findings may also be seen as suggestive of a link between epilepsy and dopamine insufficiency. A previous ${ }^{18}$ F-Fluoro-L-DOPA PET study has found marked decreases in ${ }^{18}$ F-Fluoro-L-DOPA uptake in the $\mathrm{SN}$ and PUT, suggesting impairment of dopamine activity in the patients with mTLE [22]. In disorders associated with subcortical iron deficiency, such as restless legs syndrome and Attention-Deficit/Hyperactivity Disorder, decreased iron in the subcortical nuclei have been shown to relate to alterations in dopamine production [30,31]. Moreover, in degenerative disorders, such as Parkinson's disease and Alzeimer's disease, increased subcortical iron levels have also been correlated with abnormal dopamine function [3-5]. Future study is needed to directly link MRI phase changes with dopamine levels in epilepsy.

Our correlation analysis showed that iron alterations in cortical structures in mTLE were negatively correlated 

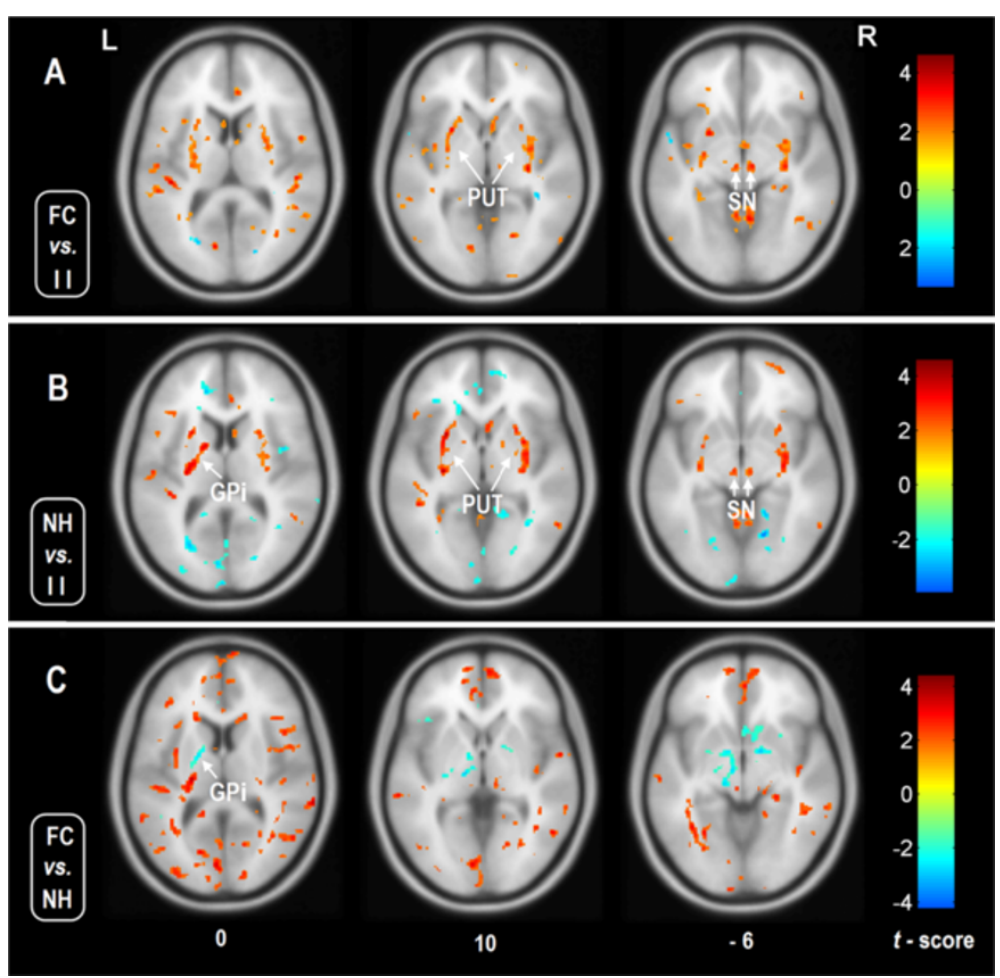

Figure 4 Comparison of phase values among the patients with precipitating factors. One-way ANOVA analysis revealed difference iron states between the patients with different pathogenesis ( $p<0.05$, FDR correction). Comparing with the subgroup of intracranial infection, the subgroups of febrile convulsion (A) and no overt history (B) both showed increased phase values, i.e., decreased iron concentration(warm color) in the PUT and SN. The difference of phase value between subgroups of FC and NH was located in the GPi (C). The results are shown by overlying on MNI 305 template image. Abbreviations: L: left; R: right; mTLE: PUT: putamen; GPi: internal globus pallidus; SN: substantia nigra; RN: red nucleus; II: intracranial infection; FC: febrile convulsion; NH: no overt history.

Table 2 Results for group comparisons of phase values

\begin{tabular}{|c|c|c|c|c|c|}
\hline Brain regions & & $\begin{array}{l}\text { mTLE vs. HC } \\
x, y, z^{*}(t)\end{array}$ & $\begin{array}{l}\text { FC vs. II } \\
x, y, z^{*}(t)\end{array}$ & $\begin{array}{l}\text { NH vs. II } \\
x, y, z^{*}(t)\end{array}$ & $\begin{array}{l}\text { FC vs. NH } \\
x, y, z^{*}(t)\end{array}$ \\
\hline \multirow[t]{5}{*}{ Corticalstructures } & L Front & $-18,44,15(-3.22)$ & None & None & None \\
\hline & R Front & $38,51,18(-4.98)$ & None & None & None \\
\hline & L Temp & $-44,13,-10(-3.63)$ & None & None & None \\
\hline & R Temp & $47,0,14(-3.87)$ & None & None & None \\
\hline & L Occip & $-11,-94,0(-4.35)$ & None & None & None \\
\hline \multirow[t]{10}{*}{ Subcortical structures } & L PUT & $-23,7,2(4.33)$ & $-25,9,1(3.55)$ & $-25,9,2(4.41)$ & None \\
\hline & R PUT & $21,5,-2(5.54)$ & $31,-19,-2(3.74)$ & $30,-17,4(3.31)$ & None \\
\hline & L GPi & $-25,-16,4(6.53)$ & None & None & None \\
\hline & R GPi & $25,-13,5(6.21)$ & None & None & None \\
\hline & L GPe & None & None & $-15,2,11(3.85)$ & $-18,-4,10(-2.71)$ \\
\hline & R GPe & None & None & None & $19,-2,10(2.72)$ \\
\hline & L SN & $-5,-20,0(4.56)$ & $-4,-19,-8(2.98)$ & $-4,-19,-9(3.47)$ & None \\
\hline & R SN & $6,-21,0(4.47)$ & $4,-20,9(3.45)$ & $4,-20,-9(3.22)$ & None \\
\hline & L RN & $-12,-7,-5(5.92)$ & None & None & None \\
\hline & R RN & $11,-3,-3(6.33)$ & None & None & None \\
\hline
\end{tabular}

Abbreviations: $m T L E$ mesial temporal lobe epilepsy, HC healthy controls, $L$ left, $R$ right, Front frontal lobe, Temp temporal lobe, Occip occipital lobe, PUT putamen, $G P i$ internal globus pallidus, GPe external globus pallidus, $S N$ substantia nigra, $R N$ red nucleus, $F C$ febrile convulsion, /l intracranial infections, $N H$ no prior history of pathogenesis. Notes: * $\mathrm{MNI}$ coordinates. 

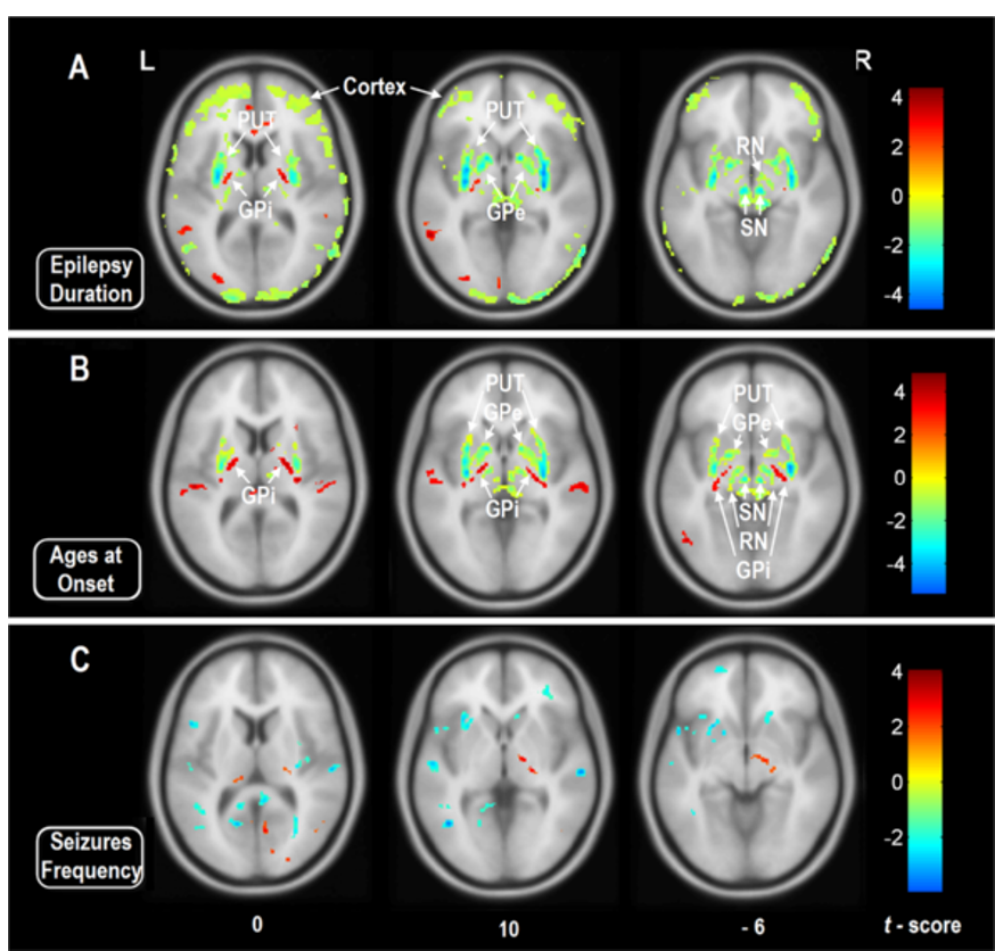

Figure 5 Voxel-based correlation analyses between the phase values and clinical variables in the patients with mTLE. Warm color denotes positive correlation, and cold color denotes negative correlation ( $p<0.05$, FDR correction). A: Correlation between phase values and epilepsy duration. The cortical and subcortical structures both showed negative correlation, denotes that the iron concentrations in these brain regions are positive correlated with epilepsy duration. B: Correlation between phase values and ages at seizures onset. Negative correlation was found between the subcortical structures including the GPe, PUT, SN and RN. In the results of A and B, the nucleus of GPi showed dissociated presence with the other subcortical nuclei. C: Correlation between phase values and seizures frequency. No meaningful region was found to have correlation. The results are shown by overlying on MNI 305 template image. Abbreviations: L: left; R: right; PUT: putamen; GPi: internal globus pallidus; GPe: external globus pallidus; SN: substantia nigra; RN: red nucleus.

with those in subcortical structures. These findings thus provide further quantitative evidence that higher cortical iron relates to lower subcortical iron, suggesting a coupled subcortico-cortical iron redistribution in MTLE. A similar pattern has been reported in a previous SWI study on laminar necrosis with hypoxia, a finding interpreted as iron being transported from ganglia to cortical structures [32]. Indeed, iron is taken up by the capillary endothelial cells from the circulation via transferrin receptors and gets sequestered in the basal ganglia [33]. The iron can also be transported from the basal ganglia along the axons to their sites of projection, where it may accumulate [33,34]. Although future studies are needed to provide more details on the exact directionality underlying our findings in epilepsy, increased cortical iron levels in mTLE may thus, at least in part, endogenously stem from subcortical structures.

\section{Alterations of brain iron and clinical relevancies}

This study investigated the relationship of several clinical factors to altered iron levels in mTLE. Although the previous basic researches have correlated the excessive iron to the cause $[8]$ or consequence $[10,14]$ of epilepsy, our study provided clinical evidence for interrogation of relationship between iron and epilepsy. Firstly, the negative correlation between the epilepsy duration and phase values indicated that the altered brain iron is related to the progression of epilepsy. Moreover, the negative correlation between the ages at seizure onset and phase values in the subcortical nuclei may indicate that the iron level in epileptic brain may be affected by the ages of seizure onset.

Subgroup comparing results among the patients suggested that the brain iron alterations might also be associated with the pathogenesis of mTLE. Patients suffering from intracranial infections showed relative high iron concentration in the PUT and RN in contrast to the other two subgroups. It is conceivable that the alteration of iron levels in intracranial infections may arise from iron-accumulation by immune cells, e.g., microglial and astrocytes are known to uptake iron more readily than neurons $[10,35]$.

\section{Methodological consideration and limitations}

SWI is a mature and widely used MRI technique that can examine the brain iron either in the form of heme 
Table 3 Correlation between phase values and clinical variables

\begin{tabular}{|c|c|c|c|c|}
\hline Brain regions & & $\begin{array}{l}\text { Duration } \\
x, y, z^{*}(t)\end{array}$ & $\begin{array}{l}\text { Onset } \\
x, y, z^{*}(t)\end{array}$ & $\begin{array}{l}\text { Seizure frequency } \\
x, y, z^{*}(t)\end{array}$ \\
\hline \multirow[t]{6}{*}{ Cortical structures } & L Front & $-33,62,13(-4.58)$ & None & None \\
\hline & R Front & $48,21,23(-4.04)$ & None & None \\
\hline & L Temp & $-36,5,-30(-4.07)$ & None & None \\
\hline & R Temp & $45,6-30(-3.23)$ & None & None \\
\hline & L Occip & $-16,-101,13(-4.47)$ & None & None \\
\hline & R Occip & $25,-101,2(-4.96)$ & None & None \\
\hline \multirow[t]{10}{*}{ Subcortical structures } & L PUT & $-30,-4,1(-6.42)$ & $-28,-11,4(-6.72)$ & None \\
\hline & R PUT & $31,-4,0(-6.32)$ & $30,-7,-5(-7.62)$ & None \\
\hline & L GPi & $-21,-10,4(3.18)$ & $-19,-8,5(3.10)$ & None \\
\hline & R GPi & $22,-8,10(3.46)$ & $19,-7,7$ (3.19) & None \\
\hline & L GPe & $-13,6,1(-5.53)$ & $-15,6,2(-5.58)$ & None \\
\hline & R GPe & $-19,2,15(-3.93)$ & $13,6,1(-5.35)$ & None \\
\hline & L SN & $-12,-11,-3(-2.72)$ & $-6,-17,-6(-5.09)$ & None \\
\hline & R SN & $15,-13,15(-3.25)$ & $7,-19,9(-5.92)$ & None \\
\hline & L RN & $-6,-19,-8(-5.38)$ & $-12,-11,-3(-4.35)$ & None \\
\hline & R RN & $7,-19,-6(-5.29)$ & $13,-11,-5(-4.73)$ & None \\
\hline
\end{tabular}

Abbreviations: $L$ left, $R$ right, Front frontal lobe, Temp temporal lobe, Occip occipital lobe, PUT putamen, GPi internal globus pallidus, GPe external globus pallidus, $S N$ substantia nigra, $R N$ red nucleus, FC febrile convulsion, /I intracranial infections, NH no prior history of pathogenesis. Notes: ${ }^{*} M N I$ coordinates.

iron, i.e., deoxyhaemoglobin, or non-heme iron, such as ferritin and haemosiderin $[7,18]$. Only non-heme iron is considered to be directly relevant to the iron metabolism [7]. Many studies have demonstrated the correlation between the SWI phase shifts and brain iron concentrations in the human brain tissue $[4,7]$. In this work, we made a few of improvements to data analysis. Firstly, we applied voxel-based analysis for phase images instead of traditional manual ROI based analysis, which can detect the statistically significant results over the whole brain with independence of any prior hypotheses. Secondly, based on a vessel segmentation, we removed components of positive phase related to veins prior to statistical analysis, a step that likely eliminated confounds related to calcium and hemodeoxyhaemoglobin [7]. In addition, although we performed a voxel-wise gray-matter volume regression in statistical analysis, the morphological effect on phase data might not be thoroughly excluded in the cortical structures. Whereas, the selective appearances of cortical changes in MRI susceptibility might indicate a pathological phenotype in epilepsy, instead of the artifact effect from cortical atrophy.

Nevertheless, this study has several limitations. Firstly, because of a diverse antiepileptic drug treatment in our patients, we could not evaluate the possible effects of antiepileptic drugs on iron alterations in mTLE [36]. Secondly, a future follow-up study is required to further clarify the relationship between the iron state and treatment outcomes, in particular with respect to seizure-freedom following surgery. Thirdly, although no patient with anaemia was found, no available data of plasma ferritin levels might be the defect of this study. Previous evidence has suggested that the status of plasma ferritin may be correlated with seizures [37,38]. Fourth, data of left-sided mTLE and matched controls were left-right flipped to produce a homogenous right-sided mTLE dataset. While this approach aimed at increasing statistical power in comparing patients to controls, it may have introduced some confounds with respect to physiological lateralization of iron deposition [25]. Finally, with the improvement of MRI technique, the most newly-developed quantitative susceptibility mapping is more accurate than SWI on assessment of brain iron content [39].

\section{Conclusion}

By measuring the phase values of the brain using susceptibility-weighted MRI, this study revealed a whole-brain pattern of iron alterations in mTLE. Besides to the anticipated finding that the cortical structures showed increased iron, we provide novel evidence showing decreased iron in the subcortical regions in mTLE. The negative correlation of iron concentrations in these two areas might suggest iron redistribution in epileptic brain. Clinical correlation analyses indicated that iron alterations are not only related to the clinical progression, 
but also to the precipitating factors. This study shed light on the pathophysiological mechanisms of iron alteration in mTLE, and provided a potential biomarker for studying epilepsy.

\section{Additional file}

Additional file 1: Figure S1. Example of ROls selections. Four ROls, including the frontal cortex (purple), GPi (green), RN (red) and SN (white) ipsilateral to the epileptogenic side, were selected according to the VBA results. Especially, the ROI drawing of the frontal cortex was carefully performed to deliberately avoid the artifact at the interface between cortex and the skull. The phase values in each ROI (drawn by 3 operators, the values were averaged) were extracted from the patients and controls. The values were compared between the patient and control groups using two-sample t tests (Table S1). Table S1. Phase values in the ROIs. Table S2. Across-subject correlation analyses of phase values seeding at the $\mathrm{RN}+\mathrm{SN}$.

\section{Competing interests}

The authors declare that they have no competing interests.

\section{Authors' contributions}

$\mathrm{ZZ}$ carried out the imaging studies, participated in the data processing and analysis, and drafted the manuscript. WL carried out the data processing and statistical analysis. BB participated in the statistical analysis and drafted the manuscript. ZW, KS and FY participated in the design of the study and data collection. $\mathrm{YL}$ and $\mathrm{GL}$ participated in the design of the study, and coordination and helped to draft the manuscript. All authors read and approved the final manuscript.

\section{Acknowledgements}

This research was supported by the Natural Science Foundation of China (Grant nos. 81422022, 81271553, 81201155, 81171328, 61131003), The National Key Technology R\&D Program of China (No. 2014BAI04B05).

\section{Author details}

'Department of Medical Imaging, Jinling Hospital, Nanjing University School of Medicine, 305\# Eastern Zhongshan Rd, Nanjing 210002, China. ${ }^{2}$ Center for Cognition and Brain Disorders, Affiliated Hospital of Hangzhou Normal University, Hangzhou 310015, China. ${ }^{3}$ Department of Social Neuroscience, Max Planck Institute for Human Cognitive and Brain Science, Leipzig, Germany. ${ }^{4}$ Department of Neurosurgery, Jinling Hospital, Nanjing University School of Medicine, Nanjing 210002, China. ${ }^{5}$ Department of Neurology, Jinling Hospital, Nanjing University School of Medicine, Nanjing 210002, China. ${ }^{6}$ Department of Psychiatry and Neuroscience, University of Florida, Gainesville, FI, USA.

Received: 19 May 2014 Accepted: 7 October 2014

Published online: 21 November 2014

\section{References}

1. Fleming RE, Ponka P: Iron overload in human disease. N Engl J Med 2012, 366(4):348-359.

2. Ke Y, Ming Qian Z: Iron misregulation in the brain: a primary cause of neurodegenerative disorders. Lancet Neurol 2003, 2(4):246-253.

3. Zecca L, Youdim MB, Riederer P, Connor JR, Crichton RR: Iron, brain ageing and neurodegenerative disorders. Nat Rev Neurosci 2004, 5(11):863-873.

4. Jin L, Wang J, Zhao L, Jin H, Fei G, Zhang Y, Zeng M, Zhong C: Decreased serum ceruloplasmin levels characteristically aggravate nigral iron deposition in Parkinson's disease. Brain 2011, 134(Pt 1):50-58.

5. Zhu WZ, Zhong WD, Wang W, Zhan CJ, Wang CY, Qi JP, Wang JZ, Lei T: Quantitative MR phase-corrected imaging to investigate increased brain iron deposition of patients with Alzheimer disease. Radiology 2009, 253(2):497-504.

6. Allen R: Dopamine and iron in the pathophysiology of restless legs syndrome (RLS). Sleep Med 2004, 5(4):385-391.
7. Haacke EM, Cheng NY, House MJ, Liu Q, Neelavalli J, Ogg RJ, Khan A, Ayaz $M$, Kirsch W, Obenaus A: Imaging iron stores in the brain using magnetic resonance imaging. Magn Reson Imaging 2005, 23(1):1-25.

8. Ong W-Y: Iron and epilepsy. In Metal lons and Neurodegenerative disorders. 1st edition. Edited by Zatta P. Singapore: World Scientific Publishing, 2003; 2004:363-397.

9. Raol YH, Brooks-Kayal AR: Experimental models of seizures and epilepsies. Prog Mol Biol Transl Sci 2012, 105:57-82.

10. Gorter JA, Mesquita AR, van Vliet EA, da Silva FH, Aronica E: Increased expression of ferritin, an iron-storage protein, in specific regions of the parahippocampal cortex of epileptic rats. Epilepsia 2005, 46(9):1371-1379.

11. Kabuto H, Yokoi I, Habu H, Willmore L, Mori A, Ogawa N: Reduction in nitric oxide synthase activity with development of an epileptogenic focus induced by ferric chloride in the rat brain. Epilepsy Res 1996, 25(2):65-68.

12. Agrawal A, Timothy J, Pandit L, Manju M: Post-traumatic epilepsy: an overview. Clin Neurol Neurosurg 2006, 108(5):433-439.

13. Herman ST: Epilepsy after brain insult: targeting epileptogenesis. Neurology 2002, 59(9 Suppl 5):S21-S26.

14. van Vliet EA, da Costa AS, Redeker S, van Schaik R, Aronica E, Gorter JA: Blood-brain barrier leakage may lead to progression of temporal lobe epilepsy. Brain 2007, 130(Pt 2):521-534.

15. Koeppen AH: The history of iron in the brain. J Neurol Sci 1995, 134(Suppl):1-9.

16. Brem F, Hirt AM, Winklhofer M, Frei $K$, Yonekawa $Y$, Wieser HG, Dobson J: Magnetic iron compounds in the human brain: a comparison of tumour and hippocampal tissue. J R Soc Interface 2006, 3(11):833-841.

17. Schultheiss-Grassi PP, Dobson J: Magnetic analysis of human brain tissue. Biometals 1999, 12(1):67-72.

18. Haacke EM, Xu Y, Cheng YC, Reichenbach JR: Susceptibility weighted imaging (SWI). Magn Reson Med 2004, 52(3):612-618.

19. Mittal S, Wu Z, Neelavalli J, Haacke EM: Susceptibility-weighted imaging: technical aspects and clinical applications, part 2. AJNR Am J Neuroradiol 2009, 30(2):232-252

20. Wriggelsworth JM, Baum H: Iron-dependent enzymes in the brain. In Brain Iron: Neurochemical and Behavioral Aspects. Edited by Youdim MBH. New York: Taylor and Francis; 1988:25-66.

21. Norden AD, Blumenfeld $\mathrm{H}$ : The role of subcortical structures in human epilepsy. Epilepsy Behav 2002, 3(3):219-231.

22. Bouilleret V, Semah F, Biraben A, Taussig D, Chassoux F, Syrota A, Ribeiro MJ: Involvement of the basal ganglia in refractory epilepsy: an 18 F-fluoro-L-DOPA PET study using 2 methods of analysis. J Nucl Med 2005, 46(3):540-547.

23. Zuo XN, Di Martino A, Kelly C, Shehzad ZE, Gee DG, Klein DF, Castellanos FX, Biswal BB, Milham MP: The oscillating brain: complex and reliable. Neuroimage 2010, 49(2):1432-1445.

24. Lerch JP, Worsley K, Shaw WP, Greenstein DK, Lenroot RK, Giedd J, Evans AC: Mapping anatomical correlations across cerebral cortex (MACACC) using cortical thickness from MRI. Neuroimage 2006, 31(3):993-1003.

25. Xu X, Wang Q, Zhang M: Age, gender, and hemispheric differences in iron deposition in the human brain: an in vivo MRI study. Neuroimage 2008, 40(1):35-42.

26. Benjamini $Y$, Hochberg $Y$ : Controlling the false discovery rate: a practical and powerful approach to multiple testing. J R Stat Soc Series B 1995, 57:289-300.

27. Ortega R, Cloetens P, Deves G, Carmona A, Bohic S: Iron storage within dopamine neurovesicles revealed by chemical nano-imaging. PLoS One 2007, 2(9):e925.

28. Depaulis A, Moshe SL: The basal ganglia and the epilepsies: translating experimental concepts to new therapies. Epileptic Disord 2002, 4(Suppl 3):S7-S8

29. Thornton R, Vulliemoz S, Rodionov R, Carmichael DW, Chaudhary UJ, Diehl B, Laufs H, Vollmar C, McEvoy AW, Walker MC, Bartolomei F, Guye M, Chauvel P, Duncan JS, Lemieux L: Epileptic networks in focal cortical dysplasia revealed using electroencephalography-functional magnetic resonance imaging. Ann Neurol 2011, 70(5):822-837.

30. Mizuno S, Mihara T, Miyaoka T, Inagaki T, Horiguchi J: CSF iron, ferritin and transferrin levels in restless legs syndrome. J Sleep Res 2005, 14(1):43-47.

31. Konofal E, Lecendreux M, Deron J, Marchand M, Cortese S, Zaim M, Mouren MC, Arnulf I: Effects of iron supplementation on attention deficit hyperactivity disorder in children. Pediatr Neurol 2008, 38(1):20-26. 
32. Kesavadas C, Santhosh K, Thomas B, Gupta AK, Kapilamoorthy TR, Bodhey N, Pendharker H, Patro S: Signal changes in cortical laminar necrosis-evidence from susceptibility-weighted magnetic resonance imaging. Neuroradiology 2009, 51(5):293-298.

33. Stankiewicz J, Panter SS, Neema M, Arora A, Batt CE, Bakshi R: Iron in chronic brain disorders: imaging and neurotherapeutic implications. Neurotherapeutics 2007, 4(3):371-386.

34. Dietrich RB, Bradley WG Jr: Iron accumulation in the basal ganglia following severe ischemic-anoxic insults in children. Radiology 1988, 168(1):203-206.

35. Vezzani A, French J, Bartfai T, Baram TZ: The role of inflammation in epilepsy. Nat Rev Neurol 2011, 7(1):31-40.

36. Garzon P, Garcia-Lopez PM, Garcia-Estrada J, Almodovar-Cuevas C, NavarroRuiz A, Roman-Maldonado S: Iron binding to nutrients containing fiber and phenytoin. Gen Pharmacol 1986, 17(6):661-664.

37. Ikeda M: Iron overload without the $\mathrm{C} 282 \mathrm{Y}$ mutation in patients with epilepsy. J Neurol Neurosurg Psychiatry 2001, 70(4):551-553.

38. Daoud AS, Batieha A, Abu-Ekteish F, Gharaibeh N, Ajlouni S, Hijazi S: Iron status: a possible risk factor for the first febrile seizure. Epilepsia 2002, 43(7):740-743

39. Bilgic B, Pfefferbaum A, Rohlfing T, Sullivan EV, Adalsteinsson E: MRI estimates of brain iron concentration in normal aging using quantitative susceptibility mapping. Neuroimage 2012, 59(3):2625-2635.

doi:10.1186/s12868-014-0117-3

Cite this article as: Zhang et al:: Brain iron redistribution in mesial temporal lobe epilepsy: a susceptibility-weighted magnetic resonance imaging study. BMC Neuroscience 2014 15:117

\section{Submit your next manuscript to BioMed Central and take full advantage of:}

- Convenient online submission

- Thorough peer review

- No space constraints or color figure charges

- Immediate publication on acceptance

- Inclusion in PubMed, CAS, Scopus and Google Scholar

- Research which is freely available for redistribution 